\title{
Using resonance acoustic emission vibrations as a method for implant insertion assessment in total hip arthroplasty
}

\author{
Jonathan CJ Wei ${ }^{1, *}$, Willem HA Crezee ${ }^{1}$, Hilda Jongeneel ${ }^{1}$, Tobias SA De Haas ${ }^{1}$, Wesley LA Kool ${ }^{1}$, Bryan J Blaauw \\ ${ }^{2}$, Jenny Dankelman ${ }^{1}$ and Tim Horeman ${ }^{1, *}$ \\ 1 Department of BioMechanical Engineering, Faculty of Mechanical, Materials and Maritime Engineering, \\ Delft University of Technology, 2628 CD Delft, The Netherlands \\ 2 Department of Orthopaedics, Elkerliek Hospital, 5707 HA Helmond, The Netherlands \\ * Correspondence: j.c.wei@tudelft.nl, t.horeman@tudelft.nl
}

\begin{abstract}
The success of total hip arthroplasty depends on the experience of the surgeon, and one of the ways the surgeon currently determines the final implant insertion depth is to listen to the change in audible pitch of the hammering sound. We investigated the use of acoustic vibration emissions as a novel method for insertion quality assessment. A non-invasive contact microphonebased measurement system for insertion depth estimation, fixation and fracture detection was developed using a simplified in vitro bone/implant $(n=5) .2583$ audio recordings were analysed in vitro to obtain energy spectral density functions. Out of the four main resonant peaks under in vitro conditions, broach insertion depth statistically correlates to increasing $3^{\text {rd }}$ and $4^{\text {th }}$ peak frequencies. Degree of fixation was also observed as higher goodness of fit (0.26-0.78 vs. 0.12-0.51 between two broach sizes, the latter undersized). Finally, however, the moment of fracture could not be predicted. A cadaveric in situ pilot study suggests comparable resonant frequencies in the same order of magnitudes with the bone model. Further understanding of the signal patterns are needed for an early warning system diagnostic system for imminent fractures, bone damage, improving accuracy and quality of future procedures.
\end{abstract}

Keywords: Medical device; surgery; hip arthroplasty; acoustics; vibration emissions

\section{Introduction}

An ever-increasing number of people are receiving total hip arthroplasties (THAs) in recent years. In the Netherlands, this number grew from 23,000 to 41,000 between 2010 and 2018 [1] and is predicted to increase even further due to population growth and increase in life expectancy. When a total hip arthroplasty (THA) is performed, the head of the femur is removed and a broach is inserted into the femur by hammering, to provide a canal in which the stem can be press-fitted. Over insertion of the broach or stem may cause fractures [2] and under insertion does not provide sufficient stability to the implant. To prevent this, it is helpful to detect the insertion depth "sweet spot" where the fixation is sufficient and the risk of fracture is minimal, defined herein as the 'end point' of insertion. Furthermore, surgeons fine-tune this end point to achieve equal limb length by using audible pitch changes of the hammering sound, and thus the ability to control the insertion distance would yield higher accuracy [3]. Therefore, the experience of the surgeon is critical for the localisation of this end point in good relation with the overall stability of the broach [4], and the success of the surgery.

In recent years, several studies have examined what can be achieved with Acoustic Emission (AE) and vibration analysis in orthopaedics [5] for wear [8], aseptic loosening [6], microdamage to bone structures, healing, and biomechanical environment assessment with free or forced vibrations [4, 9]. These studies, however, focused on using acoustic emissions as a diagnostic tool over long periods of time to assess fixation quality and wear (sometimes through the skin of patients with THA $[7,8]$ ), rather than real-time analysis of 
implant insertion quality intraoperatively. There are, however, a few studies that explored the effects of acoustic emissions as a tool for fixation, microcracking or fracture prediction during joint replacements in various models such as animal [10] and human cadaver [9]; in vitro $[11,12]$ and in vivo setups $[3,4]$ with various degrees of success.

Several other techniques of measuring AEs were also trialled. For example, Pastrav et al. [4] studied the frequency response function (FRF) change of the stem-femur system with an electrodynamic exciter (shaker) to excite low amplitude vibrations in the stem over hammer blows to detect insertion end point. They also suggested [13] that intraoperative vibration analysis requires a more user-friendly device. Oberst et al. [9] used a microphone aimed at the hammer impaction point. Pechon et al. [10] used an acoustic emission sensor placed on the stem inserted into deer femora and placed in a loading machine (quasistatically-loaded instead of striking with a hammer). These studies suggested a correlation between acoustic emission signals and occurring damage processes. Shibunama et al. [16] used a contact microphone on the bone itself $(n=1)$ to pick up less ambient noise and suggested that their system can help determine the right stem size.

The types of bone setups for acoustic emission investigations done for THAs in present literature, include sounds directly obtained from the OR [17], cadaver bone [9], animal bone [10], artificial bone [11] or a simplified bone model [12]. To design an appropriate bone model, some bone characteristics are required (shown in Table 1). In THA fracture experiments, the ultimate tensile strength of the bone model in the transverse direction plays a considerable part in determining the fracture resistance of the material.

Table 1. Mechanical properties of the human bone [15].

\begin{tabular}{cccc}
\hline Bone material & Property & Value (MPa) & Direction \\
\hline \multirow{2}{*}{ Cortical } & Tensile strength & $80-172$ & Longitudinal \\
& & $51-56$ & Transversal \\
& Elastic modulus & $11000-20000$ & Longitudinal \\
\hline \multirow{2}{*}{ Trabecular } & Compressive strength & $0.5-50$ & \\
& Elastic modulus & $5-150$ & \\
\hline
\end{tabular}

While the above investigations appear to be promising, many have been tested in controlled settings not directly applicable to surgical settings, furthermore, it is often obstructive, with complex audio recording and processing hardware such as microphones, wiring, as well as interference from ambient sounds etc. Mulier et al. [13] emphasised that while "in vivo use of vibration analysis is possible, there is an urgent need for the development of a more user-friendly, wireless device".

Another aspect of measuring AEs to consider is that impact force while broaching is not constant since it depends on both the surgeon and the impact duration. According to Oberst et al. [9], it can vary between 1 and $19 \mathrm{kN}$, with a mode of approximately $3.5 \mathrm{kN}$. Crisman et al. [18] showed a typical impact force plot with a maximum force of $7.6 \mathrm{kN}$, decreasing to zero in approximately $1 \mathrm{~ms}$. Combing these results, by integrating over time, the impact energy, which is probably more consistent than the impact force, is between $0.5-9.5 \mathrm{~J}$ per hammer blow.

The aim of the study is to develop and validate a simple, non-invasive vibration measurement system as a potential tool for estimating insertion depth, fixation, and fracture development, that does not jeopardise the current workflow. This novel, non-invasive Broach Insertion Measurement System (BIMS) is designed and tested in vitro by analysing the frequency spectrum of the hammering impact sounds in a systematic way.

With BIMS, we test the following hypotheses:

1. The cumulative energy exerted on the broach or a change in measured vibration frequency is linked to the insertion depth in the bone model.

2. The degree of fixation of the broach in the bone affects the frequencies of the hammer impact sound. 
3. The frequencies of the hammer impact sound can be used to predict imminent fracture.

\section{Materials and methods}

\subsection{Broach Insertion Measurement System (BIMS)}

\subsubsection{Microphone selection}

In order to analyse the vibration produced by hammering, the sound was recorded with a piezo contact microphone (Oyster S/S, Schaller, Postbauwer-Heng, Germany) placed directly on the broach handle. The piezo element is capable of picking up frequencies in the audible spectrum. The microphone was attached to the handle with doublesided tape (double-sided filmic tape, Tesa, Norderstedt, Germany), just above the place where the surgeon holds the handle, shown in Figure 1 (a). This enables the signal to be directly transmitted without having to travel through air.

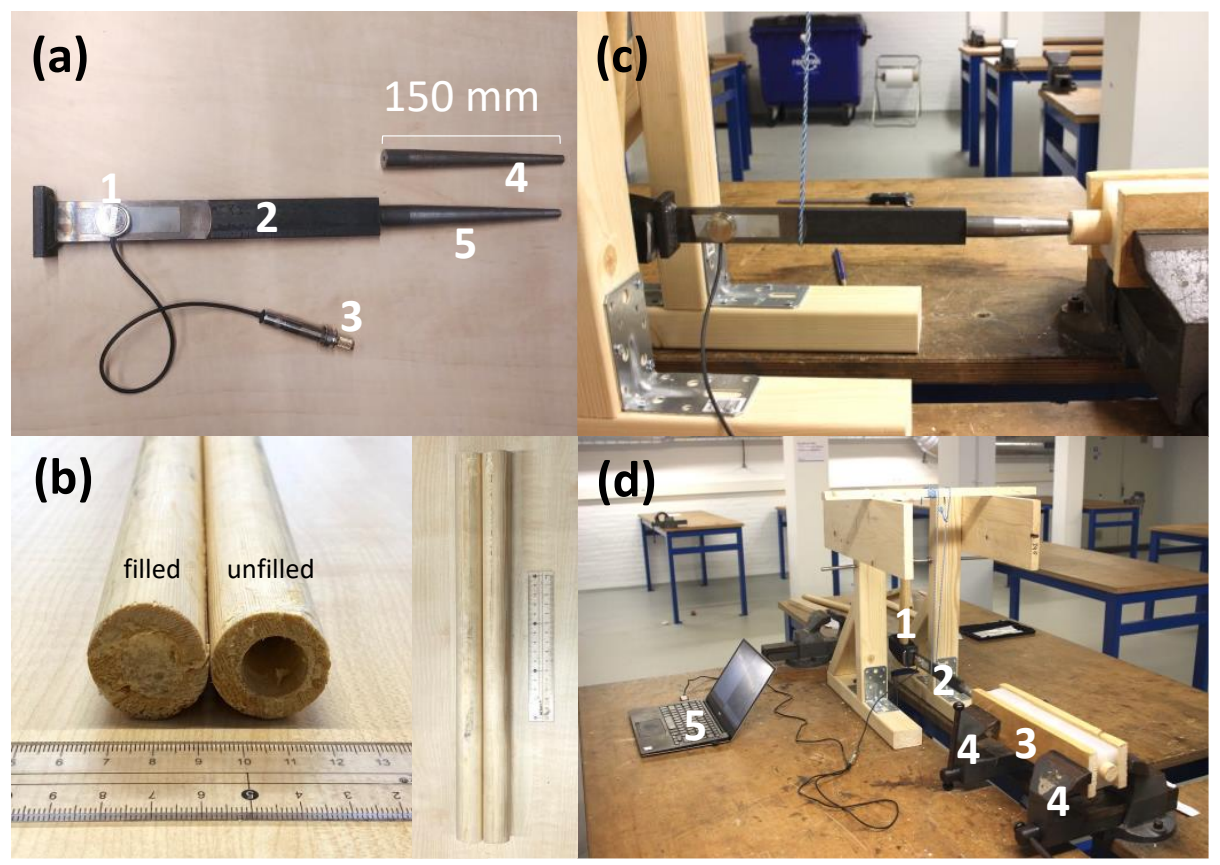

Figure 1. (a) The simplified implant model consisting of a contact microphone 1, broach handle 2, a $6.3 \mathrm{~mm}$ jack to the computer 3, smaller broach 4 and a larger broach 5. (b) Example of filled and unfilled bone model. Experiment setup (c) side view and overview (d) showing the swing hammer $\mathbf{1}$, hammer and broach model with the attached microphone 2 , bone in silicone frame 3 clamped between two vices 4 and the computer to record and process the data 5 .

\subsubsection{Audio recording}

The analogue audio signal was sampled in a USB audio adapter (EW3751 R1, Ewent, Geleen, The Netherlands) with a sampling frequency range of up to $33100 \mathrm{~Hz}$ (human audible spectrum $\sim 20 \mathrm{~Hz}-20 \mathrm{kHz}$ ) [25] and was connected to a laptop (XPS 13 9350, Dell, Round Rock TX, USA). The audio signal was recorded with Audacity (version 2.3.2, The Audacity Team) in WAV-file format, with microphone sensitivity set to $20 \%$.

\subsubsection{Data analysis}

Data was analysed using Matlab (version R2019b, MathWorks, Natick MA, USA). The WAV audio format has a unit that is relative to the maximum measurable intensity, which is set to 1 . The sample WAV file was split into separate hammer blows, from which the Fast Fourier Transform (FFT) and subsequently the Energy Spectral Density (ESD) were calculated. The frequency peaks and their energy were analysed, as well as the insertion depth and cumulative energy, defined as the cumulative hammer energy added to the system during the experiment. A typical hammer blow in time and frequency 
domain is presented in Figure $2(a-b)$. Statistical significance in correlating peak frequency and insertion depth was performed in GraphPad Prism (Prism 8, San Diego CA, USA) using a simple linear regression curve fit.

(a)

(c)
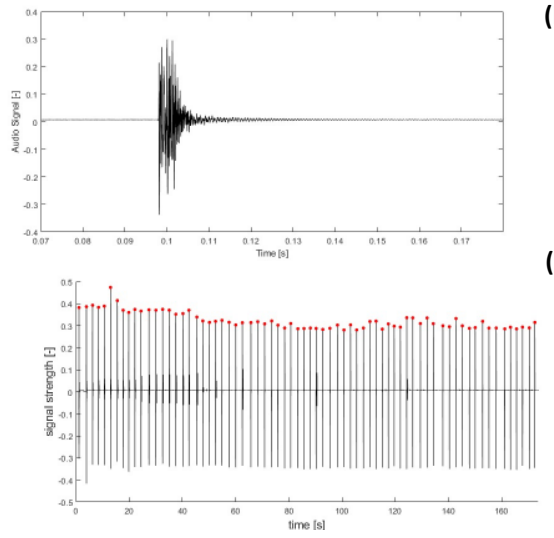

(b)

(d)
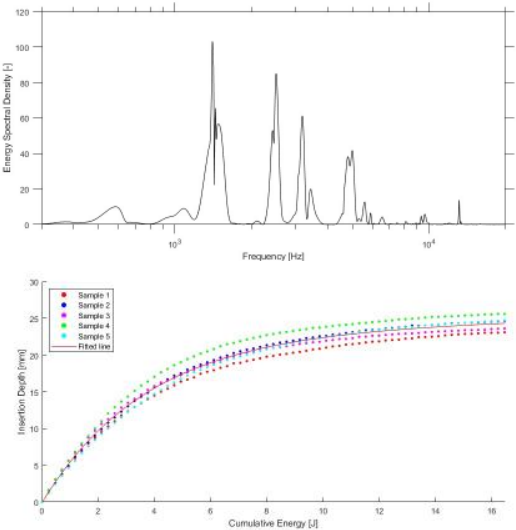

Figure 2. Typical hammer blow in time and frequency domain. (a) is the original audio signal and (b) is the signal converted to ESD. (c) A complete set of hammer blows in signal intensity over time. (d) Shows the cumulative energy over the insertion depth, caused by the hammer for the larger broach, well-fixated condition.

\subsection{In vitro experiments}

To test the BIMS and its working principle, a simplified bone model and implant model consisting of a simplified broach and connected handle were made.

\subsubsection{Custom Implant model}

The implant model consists of a broach and handle shown in Figure 1 (a). The steel (Blankstaal S235JRG2C+C/SH $18 \mathrm{~mm}$ rond, Kloeckner Metals ODS Nederland, Barendrecht, The Netherlands) broach was simplified as a round wedge with a tip diameter of $7 \mathrm{~mm}$, gradually increasing until $18 \mathrm{~mm}$ over $130 \mathrm{~mm}$ from the tip. Another thinner broach was also made, increasing from $7 \mathrm{~mm}$ to $15 \mathrm{~mm}$ over $130 \mathrm{~mm}$ to obtain an undersized condition with less fixation than the thicker broach at the same insertion depth. The steel (Platstaf 30x8 mm S 235 JR G2, Kloeckner Metals ODS Nederland, Barendrecht, The Netherlands) handle was simplified as two flat bars with a cross-section of 30x8 welded together perpendicularly, giving a total length of $255 \mathrm{~mm}$. The broach and handle are connected with M4 threading.

\subsubsection{Custom bone model:}

The dimensions of the bone were comparable to a typical femur [20]. The bone model in Figure 1 (b) was made from two materials: the cortical bone of the femur was modelled as rod of pine (Pinus sylvestris) wood (Grenen ronde stock 70\% PEFC 28x2700, Pontmeyer, Zaandam, The Netherlands) with a length of $400 \mathrm{~mm}$ and an outer diameter of 28 $\mathrm{mm}$. In this rod, a hole with a diameter of $15 \mathrm{~mm}$ and a depth of $200 \mathrm{~mm}$ was made and filled with polyurethane (PUR) foam (Precit montageschuim 30, Hornbach Baumarkt AG, Bornheim, Germany) to represent the trabecular bone. Properties of the materials used in the bone model can be found in Table 2 . The PUR foam was sprayed into the cavity until it was filled to the top and let set for a duration of at least 14 hours. A hole of $100 \mathrm{~mm}$ deep and a diameter of $8.5 \mathrm{~mm}$ was reamed in the PUR foam to model the medullary cavity and to guide the broach into the foam. A set of 5 bones per experimental group was produced. The bone model was clamped in a silicone (SR1040 Siloconen gietrubber Platinum 40, Resion Resin Technology, Moordrecht, The Netherlands) frame, representing the soft tissue of the upper leg. This frame was secured in a vice to prevent movement.

Table 2. Mechanical properties of the bone model [19].

\begin{tabular}{llll}
\hline Material & Property & Value (MPa) & Direction \\
\hline
\end{tabular}




\begin{tabular}{cccc}
\hline \multirow{2}{*}{$\begin{array}{c}\text { Pine wood (Pinus } \\
\text { sylvestris) }\end{array}$} & Tensile strength & $92-112$ & Longitudinal \\
& Elastic modulus & $2.6-3.2$ & Transversal \\
& Compressive strength & $0.03-13$ & Longitudinal \\
\hline \multirow{2}{*}{ PUR foam } & Elastic modulus & $0.33-665$ & \\
& &
\end{tabular}

\subsubsection{Experiment setup:}

The setup is shown in Figure 1 (c-d), consisting of the microphone attached to the handle, the implant model inserted into the bone model, the bone model clamped with two vices and a swing hammer that hits the handle top. The vice was tightened until the bone model did not move visibly while hammering. The starting insertion depth was 65 $\mathrm{mm}$, as the broach got to this depth without resistance. The hammer (Vuishamer $1500 \mathrm{~g}$, Picard, Wuppertal, Germany) weighed $1.5 \mathrm{~kg}$ and could be released from several fixed positions to keep the exerted energy constant. A digital calliper was used to measure the insertion depth. The force required for removing the broach was measured with a Newton meter.

\subsection{Experiments}

To determine if there is a difference in frequency spectra between an intact fixated model (good fit), an intact loose model (non-optimum, slightly undersized broach), and a broken fixated model (crack/damage in bone), three experiments were conducted on the set of five identical custom bone models. Testing was done in three experiments: an insertion experiment, a fixation experiment, and a fracture experiment.

\subsubsection{Insertion experiment}

The larger-sized broach was pushed into the bone model for $65 \mathrm{~mm}$ first, before hammer blows were applied. The swing hammer was set to apply an energy of approximately $0.24 \mathrm{~J}$ per blow (neglecting friction in the hinge and other losses) by swinging from a relative height of $16 \mathrm{~mm}$. This amount was predetermined not to cause fractures. In testing, the number of hammer blows needed for the insertion depth to change less than $0.1 \mathrm{~mm}$ per hammer blow in five consecutive blows was determined to be approximately 70 . This number was used for the insertion experiment in all samples. At this point, we consider the broach fixated. After each hammer blow, the insertion depth was measured. After the last hammer blow, the extraction force to pull out the broach from the bone model in the axial direction was measured.

\subsubsection{Fixation experiment}

The smaller, undersized broach with a tapered end was used. Testing showed that after approximately 20 hammer blows, the smaller broach reached the same insertion depth as the previous, larger-sized broach. Therefore, 20 blows are applied for the fixation experiment and again, the extraction force was measured.

\subsubsection{Fracture experiment}

A fracture with a length of $50 \mathrm{~mm}$ and $1 \mathrm{~mm}$ width was sawn in each of the five bone models. This length was chosen after fracturing several bone models and an average fracture length was obtained. The large broach was used for this experiment. Testing showed that the final insertion depth was reached after approximately 100 hammer blows and this number was used in the fracture experiment. Another set of five bone models (without a pre-sawn crack) were used to compare fracture by hammering until fracture occurs. If no fracture occurred, then the energies were increased until an approximate $1.9 \mathrm{~J}$ maximum by dropping the hammer from a relative height of $132 \mathrm{~mm}$.

\subsection{Validation experiment}

To demonstrate the appropriateness of this setup in clinical settings, we also performed a validation experiment on a cadaver hip arthroplasty during a training session. The study was carried out in Erasmus Medical Centre, Rotterdam, The Netherlands. The 
acoustic microphone was attached to the surgical handle of the Corail implant (Depuy Synthes, Johnson\&Johnson, Raynham MA, USA).

\subsection{Frequency estimation}

\subsubsection{Eigen Frequency}

The simplified model allowed an analytical estimation of the eigen frequencies and frequency shifts to help interpret and compare the measured results. For these calculations, the implant model was simplified as a rectangular beam with consistent cross-section $(30 \times 8 \mathrm{~mm})$ and clamped - free boundary conditions. The implant model length $L$ from the broach handle was $350 \mathrm{~mm}$ at the starting point $s$ and $325 \mathrm{~mm}$ at the end point $e$. The implant model material was modelled as steel with a Young's modulus $E$ of $210 \mathrm{MPa}$ and a density $\rho$ of $7850 \mathrm{~kg} / \mathrm{m}^{3}$. The eigen frequencies $\omega$ per mode $i$ for a clamped - free beam, with an area moment of inertia $L$ and mass per unit length $\mu$ is calculated by [21]:

$$
\omega_{i}=\left(\left(i=\frac{1}{2}\right) \pi\right)^{2} \frac{1}{L^{2}} \sqrt{\frac{E I}{\mu}}
$$

Equation 1

The Eigen frequencies were calculated for the first four modes in the direction of the width and height of the bean, defined as the $\mathrm{x}$ - and y-direction, respectively, are shown in Table 3. The height of these peaks is defined as the peak energy and their frequencies as the peak frequency. The cumulative energy is defined as the sum of the hammering energy added to the system.

Table 3. Analytical estimations of the eigen frequencies of the broach at the starting and ending positions in $\mathrm{x}$ and $\mathrm{y}$ directions.

\begin{tabular}{lllll}
\hline Mode $\boldsymbol{i}$ & $\boldsymbol{\omega}_{\mathrm{s}, \mathrm{x}, \mathrm{i}}(\mathrm{Hz})$ & $\boldsymbol{\omega}_{\mathrm{s}, \mathrm{y}, \mathrm{i}}(\mathrm{Hz})$ & $\boldsymbol{\omega}_{\mathrm{e}, \mathrm{x}, \mathrm{i}}(\mathrm{Hz})$ & $\boldsymbol{\omega}_{\mathrm{e}, \mathrm{y}, \mathrm{i}}(\mathrm{Hz})$ \\
\hline $\mathbf{1}$ & $2.41 \cdot 10^{2}$ & $9.02 \cdot 10^{2}$ & $2.79 \cdot 10^{2}$ & $1.05 \cdot 10^{3}$ \\
$\mathbf{2}$ & $2.17 \cdot 10^{3}$ & $8.12 \cdot 10^{3}$ & $2.51 \cdot 10^{3}$ & $9.42 \cdot 10^{3}$ \\
$\mathbf{3}$ & $6.01 \cdot 10^{3}$ & $2.26 \cdot 10^{4}$ & $6.98 \cdot 10^{3}$ & $2.62 \cdot 10^{4}$ \\
$\mathbf{4}$ & $1.18 \cdot 10^{4}$ & $4.42 \cdot 10^{4}$ & $1.37 \cdot 10^{4}$ & $5.13 \cdot 10^{4}$ \\
\hline
\end{tabular}

\subsubsection{Statistical analyses}

Firstly, a simple linear regression analysis was performed at each frequency peak to determine whether there is a statistically significant increase in the Eigen frequencies with insertion depth using Prism 8 statistical package (GraphPad, San Diego CA, USA). Where results show significant non-zero slopes of $\mathrm{p}<0.05$ are considered as a (increasing) shift. Secondly, an exponential growth nonlinear regression analysis was performed on peak energy values to determine the 'convergence' of the data, as an indicator of implant fixation. The $\mathrm{R}^{2}$ "goodness of fit" coefficient was used as a quantifier of the scatter (higher value implies higher degree of fixation). Analysis was also performed with Prism 8.

\section{Results}

In this paper, we investigated the acoustic emissions caused by hammering on the hip replacement broach handle. Three experiments were performed to investigate the effect of AEs from broach insertion characteristics, the fixation quality with a larger and smaller broach, and fractured samples.

\subsection{General observations}

When hammered into the bone model, the simplified implant model produced frequency bands centred around four main resonant peaks, namely $1400 \mathrm{~Hz}, 2500 \mathrm{~Hz}, 3150$ $\mathrm{Hz}$ and $4700 \mathrm{~Hz}$, with a width of approximately $300 \mathrm{~Hz}$. A typical hammer blow in raw audio signal is presented in Figure 2 (a) in the time domain and (b) the frequency domain, and a whole set of hammer blows is shown in Figure 2 (c).

\subsection{Energy spectral density}


The analysed ESD plots shown in Figure 3 compare the in vitro bench top setup (a-c) versus an in situ cadaver study (d). The main observable peaks appeared to be consistent with each other, in terms of locations, although the peak energy heights of the non-fixated samples (b) are much greater than the well fixated group. In terms of determining fracture of the bone (c), it is difficult to determine (if at all), whether a fracture is present in the bone.

(a)

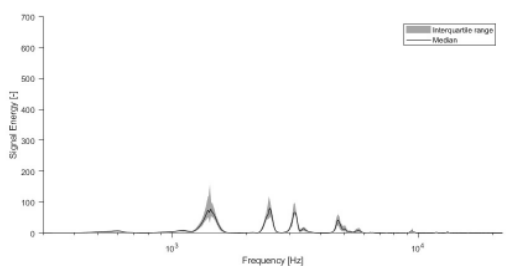

(c)

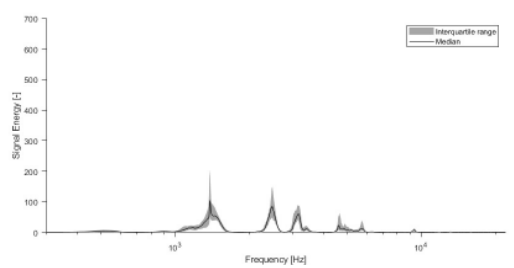

(b)

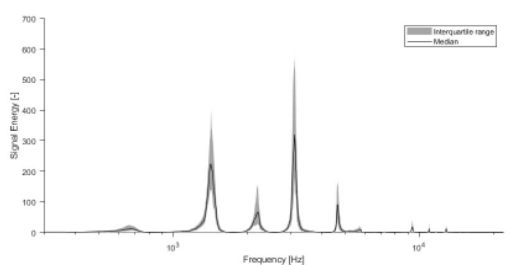

(d)

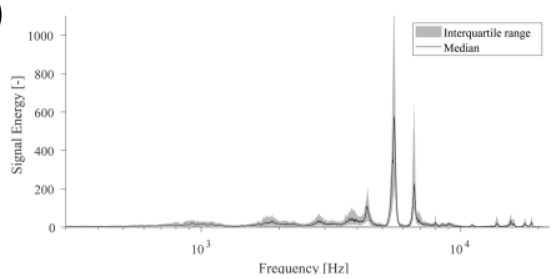

Figure 3. The median \pm interquartile range (shaded) ESD plots comparing between the (a) last 20 hammer blows of the intact samples, vs (b) the undersized/underfitted broach, the (c) fractured samples, as well as the ESD recording from a pilot cadaver study using actual Depuy Synthes Corail components shown in (d). Interquartile range is shown instead of standard deviation because the distribution was asymmetrical.

The number of significant peaks observed in the in situ study was 4, while the in situ study showed small peaks at approximately $1900 \mathrm{~Hz}, 2900 \mathrm{~Hz}, 3850 \mathrm{~Hz}, 4400 \mathrm{~Hz}, 6650 \mathrm{~Hz}$ and $15 \mathrm{kHz}$. An exceptionally high peak was observed at around $5550 \mathrm{~Hz}$. The absolute value of signal energy was also much greater for the cadaver study, presumably as noted in the experiment section that the hammer blows exerted by the surgeon is greater than the controlled hammer blows from the rig. Despite these differences, the peaks recorded from both in vitro and in situ studies appeared in the same order of magnitude (in the range of $10^{3}-10^{4} \mathrm{~Hz}$ ), even though there are differences in materials and geometry.

\subsection{Insertion experiment}

Figure 2 (d) shows a positive correlation between the insertion depth and cumulative energy - the same amount of energy is given in each hammer strike, but as can be observed, the initial insertion depth increases linearly, until approximately $10 \mathrm{~mm}$ deep, then slowly flattens to a plateau in the end. All samples follow the same pattern. It is clear in Figure 4 that all four peak energies from the ESD can be used as an indicator to predict the insertion depth for a controlled energy applied with the hammer. The $\mathrm{R}^{2}$ values of the exponential fit for resonant peaks $1-4$ are $0.31,0.78,0.70$ and 0.26 respectively (Table 5). An upwards shift in peak frequency trend (significantly non-zero slope with $\mathrm{p}<0.0004$ 0.0001 ) was observed for the $3^{\text {rd }}$ and $4^{\text {th }}$ peaks in both the smaller and larger broach, when inserted further into the bone model (Table 6). 
(a)

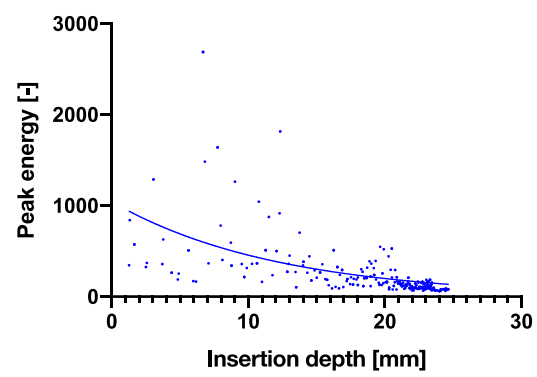

(c)

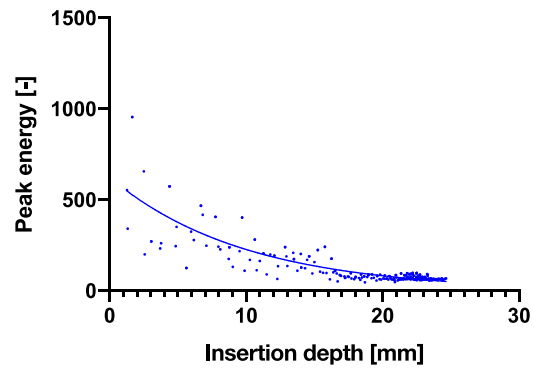

(b)

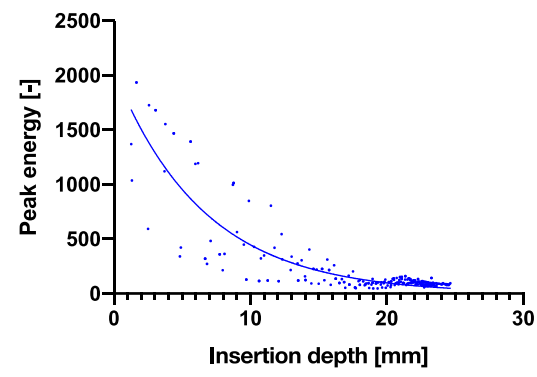

(d)

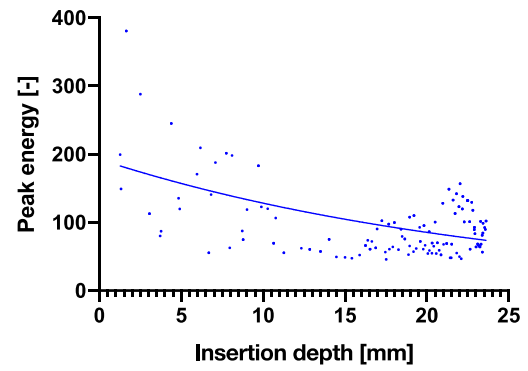

Figure 4. Capability of predicting insertion depth with frequencies by peak energy vs. insertion depth through the course of inserting the broach into the bone. (a-d) are the isolated peak energies (larger broach condition) for the four main peak frequencies at $1400 \mathrm{~Hz}, 2400 \mathrm{~Hz}, 3140 \mathrm{~Hz}$ and $4660 \mathrm{~Hz}$ respectively with an exponential trendlines. The same graphs with hammer blows on the $\mathrm{x}$-axis are shown in Supplementary Figure 3.

Table 4. Simple linear regression of Figure 7. Frequency peaks 3 and 4 for both small and large broaches show correlating increasing pitch frequency with insertion depth.

(a) F. peak (a) F. peak (a) F. peak (a) F. peak (b) F. peak (b) F. peak (b) F. peak (b) F. peak

\begin{tabular}{|c|c|c|c|c|c|c|c|c|}
\hline & 1 & 2 & 3 & 4 & 1 & 2 & 3 & 4 \\
\hline Slope & 0.1314 & 1.273 & 1.519 & 3.812 & 0.3982 & -0.2705 & 1.331 & 1.5 \\
\hline $\begin{array}{l}\text { Y-inter- } \\
\text { cept }\end{array}$ & 1420 & 2466 & 3116 & 4641 & 1423 & 2201 & 3096 & 4634 \\
\hline $\begin{array}{l}\text { X-inter- } \\
\text { cept }\end{array}$ & -10806 & -1938 & -2052 & -1218 & -3574 & 8135 & -2326 & -3090 \\
\hline 1/slope & 7.609 & 0.7858 & 0.6585 & 0.2624 & 2.511 & -3.696 & 0.7512 & 0.6667 \\
\hline R squared & 0.002108 & 0.05928 & 0.09989 & 0.1856 & 0.04461 & 0.008432 & 0.1517 & 0.1351 \\
\hline Sy.x & 17.49 & 30.97 & 27.93 & 51.12 & 20.37 & 32.61 & 34.75 & 42.06 \\
\hline $\mathrm{F}$ & 0.7329 & 21.93 & 38.06 & 59.94 & 4.529 & 0.5698 & 17.35 & 13.59 \\
\hline DFn, DFd & 1,347 & 1,348 & 1,343 & 1,263 & 1,97 & 1,67 & 1,97 & 1,87 \\
\hline$P$ value & 0.3925 & $<0.0001$ & $<0.0001$ & $<0.0001$ & 0.0359 & 0.453 & $<0.0001$ & 0.0004 \\
\hline $\begin{array}{l}\text { Deviation } \\
\text { from zero? }\end{array}$ & NS & $* * * *$ & $* * * *$ & $* * * *$ & * & NS & $* * * *$ & $* * *$ \\
\hline Equation & $\begin{array}{c}Y= \\
0.1314^{*} X+ \\
1420\end{array}$ & $\begin{array}{c}Y= \\
1.273^{*} X+ \\
2466\end{array}$ & $\begin{array}{c}Y= \\
1.519^{*} X+ \\
3116\end{array}$ & $\begin{array}{c}Y= \\
3.812^{*} X+ \\
4641\end{array}$ & $\begin{array}{c}Y= \\
0.3982 * X+ \\
1423\end{array}$ & $\begin{array}{c}Y=- \\
0.2705^{*} X+ \\
2201\end{array}$ & $\begin{array}{c}Y= \\
1.331^{*} X+ \\
3096\end{array}$ & $\begin{array}{c}Y= \\
1.500^{*} X+ \\
4634\end{array}$ \\
\hline
\end{tabular}

\subsection{Fixation experiment:}


The same experiment with the above insertion experiment was performed with a slightly smaller/thinner broach to simulate undersized implant, shown in Figure 5. The poorer fixation showed no- $(\mathrm{a}, \mathrm{c}, \mathrm{d})$ or poor convergence $(\mathrm{b})$ of the peak energies even as the stem went further into the bone model. The broach was also extracted after full insertion. The smaller broach extraction force was recorded at $60 \pm 10 \mathrm{~N}$, while the larger broach was recorded at $210 \pm 40 \mathrm{~N}$. The extraction force for the large broach due to the tighter fit is higher and is thus considered more (adequately) fixated than the smaller broach. The $\mathrm{R} 2$ values of the exponential fit for resonant peaks $1-4$ are $0.12,0.51,0.20$ and 0.17 respectively (Table 5), all lower than that of the larger broach.

(a)

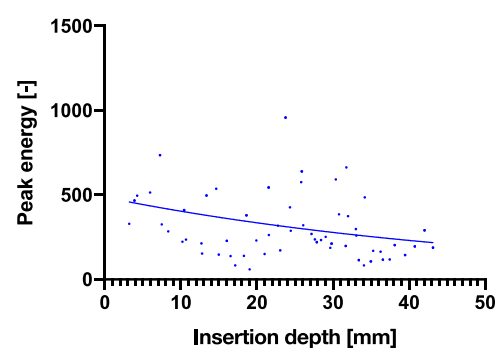

(c)

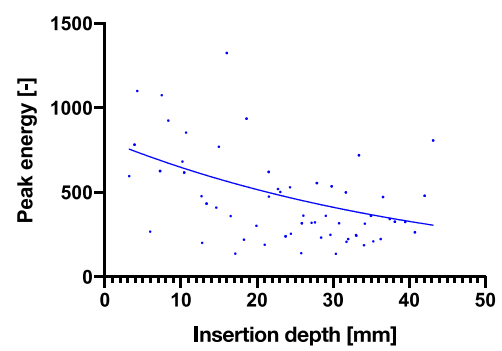

(b)

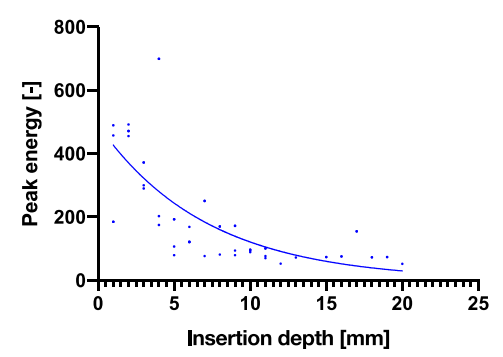

(d)

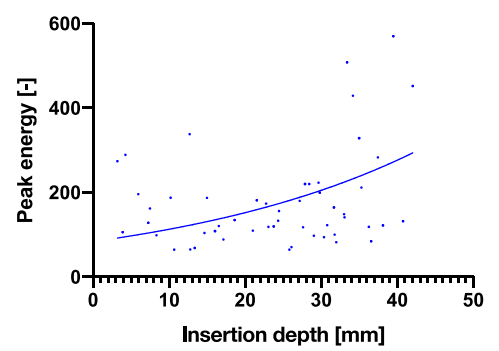

Figure 5. Capability of predicting fixation quality using the spread of data in peak energy vs. insertion depth. (a-d) are the isolated peak energies (smaller broach condition) for the four main peak frequencies at $1400 \mathrm{~Hz}, 2220 \mathrm{~Hz}, 3140 \mathrm{~Hz}$ and $4660 \mathrm{~Hz}$ respectively with exponential trendlines. The same graphs with hammer blows on the x-axis are shown in Supplementary Figure 4.

\subsection{Fracture experiment}

The deliberately created crack in the bone model appeared to show similar resonant peaks (height, width and position), in Figure 3 (a, c), and from the isolated peak energy charts in Figure 6 (a-d), they appear to show a good degree of convergence, with the exception of the fourth peak (d). This is perhaps due to the small magnitude of the peak energy as observed in Figure 3 (c). Overall, it is difficult to distinguish between the nonfractured and fractured groups. For the fractured bone condition, the $\mathrm{R}^{2}$ values of the exponential fit for resonant peaks $1-4$ are $0.00,0.44,0.36$ and 0.02 respectively (Table 5). These values are all lower compared to the larger broach; however, the $3^{\text {rd }}$ and $4^{\text {th }}$ peak fittings are higher than that of the smaller broach (Table 6). 
(a)

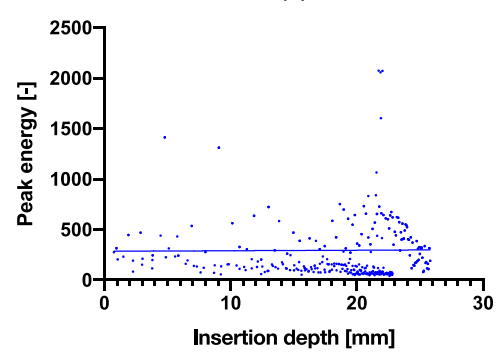

(c)

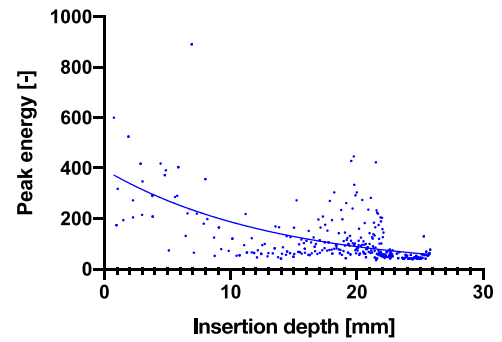

(b)

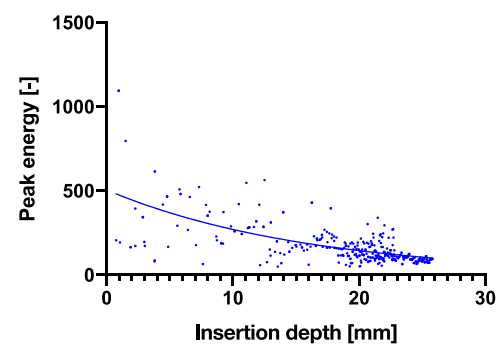

(d)

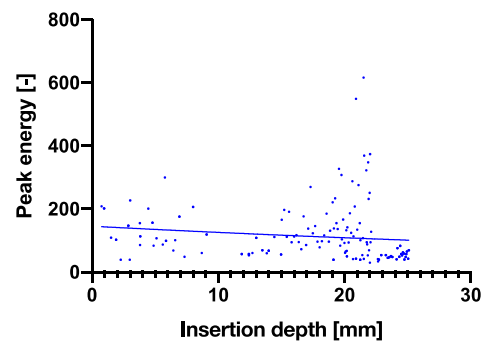

Figure 6. (a-d) are the isolated peak energies of each hammer blow, plotted as peak energy vs. insertion depth (fractured bone model condition) for the four main peak frequencies at $1400 \mathrm{~Hz}$, $2400 \mathrm{~Hz}, 3140 \mathrm{~Hz}$ and $4660 \mathrm{~Hz}$ respectively with exponential trendlines. The same graphs with hammer blows on the $\mathrm{x}$-axis are shown in Supplementary Figure 5.

\subsection{Cadaver pilot study}

To validate to which extent the results of the in vitro experiments was related to a real THA, the in situ results of the cadaver study were compared. We assumed an estimated impact energy of $1.75 \mathrm{~J}$ exerted by the surgeon, following the calculations proposed in literature [18], with the mode of $3.5 \mathrm{kN}$ as the average hammering force. While the exact insertion depth of the cadaver study was unknown, a rough approximation of $20 \mathrm{~mm}$ can be made. The broach inserted was a standard size 9, as specified in the Actis Surgical Technique [24]. 59 blows were needed to hammer the broach into its end position, giving an estimated cumulative energy of approximately $103.25 \mathrm{~J}$ - which is about an order of magnitude lower when compared to the bone model ( 16 J). Table 4 shows the details between the in vitro and the in situ trial. The ESD of the cadaver study in Figure 3 (d) show different peaks (two main peaks as opposed to four) than the in vitro models (a-c), with different spread and peak magnitudes (1-6 times greater). There were two main peaks with several smaller peaks, instead of four main peaks.

Table 5. Comparison between the in vitro bone model vs. in situ cadaver trial.

\begin{tabular}{lrr}
\hline & $\begin{array}{c}\text { Bone model insertion } \\
\text { experiment }\end{array}$ & Cadaver trial \\
\hline Hammer blows [\#] & 50 & 59 \\
Insertion depth [mm] & 23 & 20 \\
Energy per blow [J] & 0.24 & 1.75 \\
Cumulative energy [J] & 12 & 103.25 \\
Energy needed per unit length to advance broach & 0.52 & 5.16 \\
[J/mm] & & \\
\hline
\end{tabular}

\section{Discussion}


We performed experiments with two broach models, a 'normal' condition and an undersized condition, and two bone models, non-fractured and fractured. The objectives were to identify differences that could help determine the insertion depth of the broach into the bone through controlled hammering, the differences between a well-fixated and an under-fixated broach, and finally, the prediction of an imminent fracture due to over insertion and/or excessive hammering force. In general, the bone and broach models provided us with the ability to carry out the in vitro experiments consistently. In both the in vitro and in situ study, the contact microphone recorded a remarkably low amount of ambient noise, which suggests that it can be a useful tool in the OR and not just limited to recording THA acoustic emissions. In all studies, the frequency peaks are clearly visible and can be isolated to analysed, for example the peak energy. The contact microphone used is mountable on the surgical handle and the vibration is transmitted without having to travel through air. In addition, the microphone is not invasive and is easy to place. Future designs can investigate a wireless solution.

The bone model was designed this way so that batches of them can be made consistently; artificial bone (vs. animal/cadaver bone) provides more consistency; and we expect the principles of the vibrations to be comparable between the model and actual bones, although the frequencies might differ. Additionally, PUR foam shares similar structure and compressive strength with trabecular bone [15, 19]. An effort was made to source hard wood but was unavailable but overall, the material was comparable (in Table 1) to real bone. When performing a frequency analysis during testing, the material properties that determine the eigen frequency of the bone model could be considered. We assume the eigen frequency of the bone model has a minimal impact on the measured vibrations, as the contact microphone was directly connected to the broach handle.

To use the peak energies and frequency bands as a tool to measure insertion depth, this was possible by observing the gradually increasing trend in resonant frequency shift upwards - which is expected when considering this as a fixed-free cantilever beam where the effective free end becomes shorter. The upward frequency shift was visible in the third and the fourth peaks (Table 6 for significantly nonzero slopes where $p<0.05$ ), in the order of $\sim 100 \mathrm{~Hz}$ from the beginning to the end insertion over approximately $25 \mathrm{~mm}$. This increase would most likely increase further with a real broach, as the change in insertion distance is much greater. It would be possible then to predict insertion depth, if the input force/energy is known or controllable, an extrapolation could be made from the first few hammer blows. For example, taking the peak energies ( $2^{\text {nd }}$ peak) recorded in the first experiment. A logarithmic equation was obtained for Supplementary Figure 1, with insertion depth (ID) in mm approximated as: $I D=-7.7 \ln \frac{P E}{1532}$, where PE is the peak energy (Supplementary Figure 2). It is important to note that this equation is based on our bone model, and will likely differ in real life, although we believe the logarithmic trend is still valid. Other factors that may influence the insertion depth include the surgeon's hammering force and bone quality. This supports our original hypothesis that cumulative energy can be used as a parameter for insertion depth prediction. The ability to predict insertion depth would therefore increase the fitting accuracy and prevent over-insertion.

With fixation, we hypothesised that a difference is detectable between a well-fitted and an under-sized broach. The main difference identified here is that peaks 1 and 3 appeared to be greater (more than double) than that of a well-fixated broach (Figure 3 (a) at \pm 100 vs. (b) at \pm 200 - 300 , other peaks at \pm 100 ). Presumably, a looser fit dampens the vibrations less in an idealised free-fixed beam. The resonant frequencies (peaks 3 and 4) also shifted upwards significantly for both larger and smaller broaches $(p<0.0004-0.0001)$, as the broach is inserted further into the bone model (Figure 7). On the contrary, the consistency of the peak energies converged less between a poorly fixated and a well-fixated model (Figure 5 vs. Figure 4 ) as seen in the goodness of fit $\left(\mathrm{R}^{2}\right)$ between the two datasets in Table 5. This finding was previously unreported in literature and in future the $\mathrm{R}^{2}$ value could be used as an indicator/threshold to determine the fixation quality. 
(a)
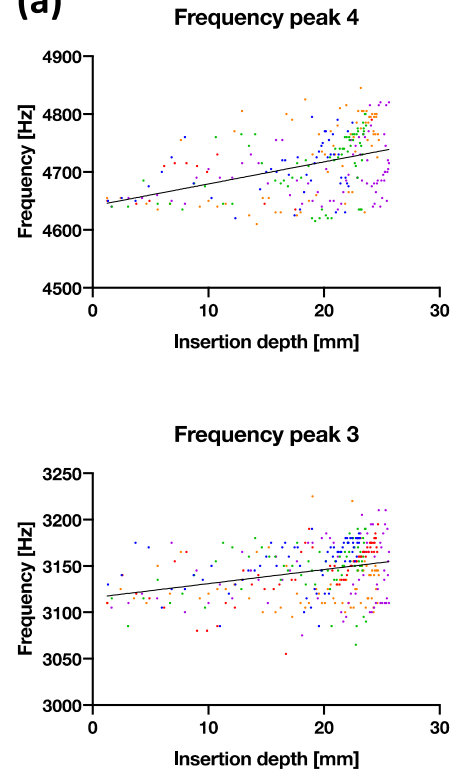

Frequency peak 2

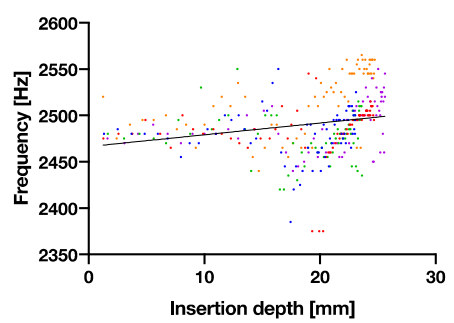

Frequency peak 1

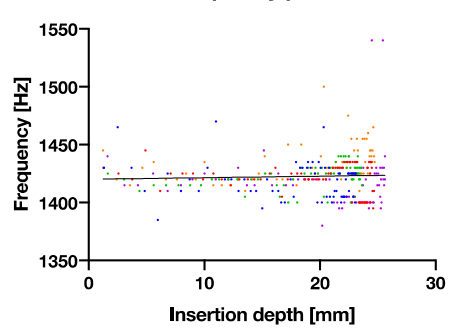

(b) Frequency peak 4

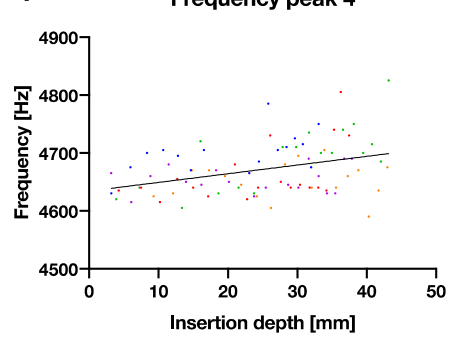

Frequency peak 3

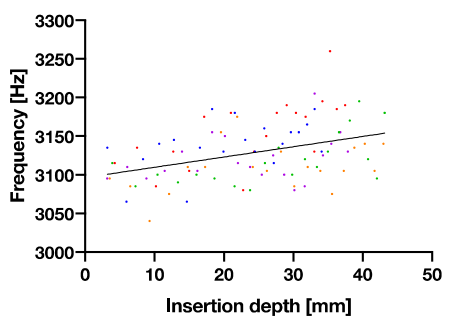

Frequency peak 2

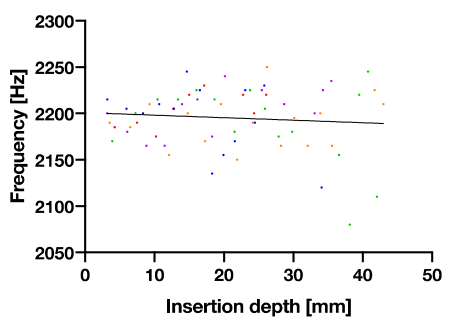

Frequency peak 1

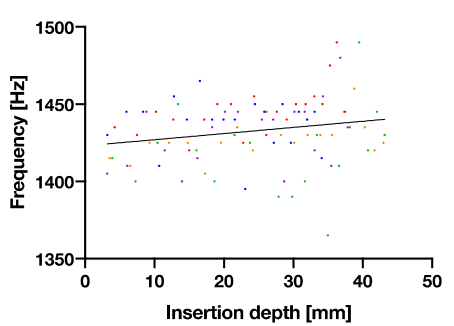

Figure 7. The correlation between recorded frequency vs. broach insertion depth with linear trendlines. (a) shows the larger broach with good fixation and (b) shows the smaller broach with poor fixation. Each row represents the four main frequency peaks observed.

Table 6. Curve-fitting of Figure 4 - Figure 6. The degree of convergence of the pitch consistency analogous to the goodness of fit $\left(\mathrm{R}^{2}\right)$ suggests fixation quality.

\begin{tabular}{ccccc}
\hline Nonlinear regression & \multicolumn{4}{c}{ Peak energy } \\
\hline $\begin{array}{c}\text { Exponential growth equation } \\
\text { Best-fit values }\end{array}$ & Figure 4. (a) & Figure 4. (b) & Figure 4. (c) & Figure 4. (d) \\
\hline Y0 & 1044 & 2041 & 624.1 & 192.6 \\
k & -0.0826 & -0.1519 & -0.1009 & -0.04061 \\
Tau & -12.11 & -6.585 & -9.913 & -24.62 \\
Doubling Time & -8.391 & -4.564 & -6.871 & -17.07 \\
R squared & 0.3103 & 0.7783 & 0.6959 & 0.2554 \\
\hline
\end{tabular}

Figure 5. (a) Figure 5. (b) Figure 5. (c) Figure 5. (d) 


\begin{tabular}{ccccc} 
Y0 & 486.7 & 491.6 & 813.7 & 83.88 \\
$\mathrm{k}$ & -0.01864 & -0.1399 & -0.02262 & 0.02983 \\
Tau & -53.65 & -7.15 & -44.21 & 33.53 \\
Doubling Time & -37.19 & -4.956 & -30.64 & 23.24 \\
R squared & 0.1199 & 0.5147 & 0.1979 & 0.1682 \\
\hline & Figure 6. (a) & Figure 6. (b) & Figure 6. (c) & Figure 6. (d) \\
\hline Y0 & 285.7 & 505.9 & 393.7 & 145.3 \\
k & 0.001743 & -0.06232 & -0.07446 & -0.0145 \\
Tau & 573.9 & -16.05 & -13.43 & -68.98 \\
Doubling Time & 397.8 & -11.12 & -9.309 & -47.82 \\
R squared & 0.0001033 & 0.4357 & 0.355 & 0.02008 \\
\hline
\end{tabular}

The peaks also appeared to differ in location and spacing, and thus, if the frequency patterns are known for a well-fixed implant, then it should be possible to identify undersized or inadequately fitted implants in a patient with an overall ESD view of the acoustic emissions. During THA, the measured values may differ from the reported values herein with the model, as human bone is harder than the wood model, the cumulative energy may be higher before the end point is reached (also depending on how the bone is reamed). The exact value of the peak energy also depends on age or bone density [23] and the measuring equipment and settings, as the amplitude of the audio file (.wav) is on a relative scale. Therefore, we suggest using a relative threshold for a more robust model to be used on clinical data.

In regards to fracture, it was difficult to predict imminent fracture in our study. A set of data is shown in Supplementary Figure 4. This is the third peak energy graph for one of the samples. It can be observed that after the initial decrease in peak energies, the energy increased slowly as the hammer blows continued to insert the stem further into the bone. This small and steady increase was not as visible as the other non-fractured groups. Whether this small increment of peak energy is an indication of stable crack growth, is so far only conjecture and needs further examination. Other cracked samples were fractured much earlier in the test, so this trend was not observed. Only immediately after fracture can an increase in peak energy and scatter be observed (most probably due to decreased fixation), but this indicator would not be suitable to prevent fracture in our setup.

A test carried out (Supplementary Figure 3) to see what it takes to crack the sample suggest that the insertion depth eventually stops advancing with each hammer blow, and that the energy of each strike has to be increased to advance the broach further, until the bone model cracks. This raises the question if a hammer force exists which is impossible to cause fractures, while achieving the desired fixation. We propose that a more realistic cracked bone model may also show different crack growth or propagation modes and whether a saw cut created herein is an appropriate representation of a fracture. Perhaps a more representative method would be to create the crack using a chisel, although the fracture length would be less consistent. For now, though, it is difficult to predict fracture. We speculate regardless that fracture risk would be higher under a well-fixated condition than an under-fixated condition.

In relation to the cadaver pilot study, the model performed well in terms of giving a similar range of peak resonant frequencies when comparing with the in situ pilot study tested in a cadaver. The cadaver study also demonstrated that the setup is suitable for use in the OR environment with minimal intrusion to the surgeon, although the data was only analysed after the session. The cumulative energy exerted by the surgeon was approximately an order of magnitude higher than that of the bone model $\left(\sim 10^{2} \mathrm{~J}\right.$ vs $\left.\sim 10^{1} \mathrm{~J}\right)$, and about ten times more energy required to move the same distance when compared with the bone model. Even though this is a very rough estimate with limited sample size, it should give an idea on how the bone models used in this research compare to real bones 
in OR conditions in terms of energies needed to insert a stem. It should be kept in mind that the way the broach handle connects to the broach may alter the acoustic emission.

Additionally, the extra time spent setting up and configuring the device would cost expensive operating room (OR) time. So, the design of such AE measurement should take usage time into consideration. Furthermore, devices that can reduce OR time add value to the surgery - in this case, measuring AEs could help inserting the implant more efficiently while also minimising the risk of bone fractures that need repairing. The use of vibration analysis in assessing broach and stem fixation and fracture detection can thus prove to be a powerful tool during THA, as well as an invaluable learning tool. As the experience of the surgeons plays a substantial role in the successful outcome of the surgery, it is necessary that they are adequately trained for this procedure. A device that gives surgeons the feedback on the position and fixation of the broach and imminent fractures could help new orthopaedic surgeons develop the experience they need to perform the surgery more quickly.

In future, this set of experiments should be carried out on real bones and conditions to gather more representative data, and to quantify the variability inherent in biological materials. The bone model was 20x less stiff than that of the human bone. The ideal "end points" in real life should also be quantified to build up a database for comparison. Hammering sound recorded in the OR should be analysed in real time to provide and objective reading for the surgeon. A simple, no-setup display of the data should also be studied. For use in actual surgeries though, the microphone setup also needs to be made wireless and sterilisable. Alternative sound recording methods could also be explored, such as with a regular microphone, however, background noise issues will need to be isolated. Regardless of the method of recording, measuring acoustic emissions from arthroplasty surgeries, including the shoulder and the knee, could eventually become a mainstream tool to analyse the quality of implantation, as well as potentially insertion, fixation and fracture prediction and prevention, forming the basis for assisted autonomous arthroplasty surgery.

\section{Conclusions}

This paper explores the possibilities of using vibration analysis in arthroplasty surgeries in a user-friendly setup. While the earlier literature suggests that vibration analysis can offer considerable benefits such as insertion depth and fracture prediction, it has never been used in a small or convenient setup suitable for the OR. Insertion depth of the broach followed a predictable pattern with a known amount of energy applied at each hammer blow. Results suggest that the third and fourth resonant frequency during a hammer blow correlated with insertion depth. The fixation experiment showed that it is possible to identify the degree of fixation based on how well the recorded energy peaks converge. The fracture experiment showed that it is difficult to predict imminent fracture with the current conditions, as the resonance peaks appeared quite similar to the group without a cut in the model. Only after cracking, the peak energy increased back to the original magnitude at the start of hammering. With further refinements to the system, such as sterilisation environment compatibility, and a display of the recorded data, it can eventually be implemented in clinical studies.

\section{Funding}

This study was funded by The Netherlands Organisation for Heath Research and Development/De Nederlandse Organisatie voor Gezondheidsonderzoek en Zorginnovatie: Innovative Medical Devices Initiative (IMDI) Technology for Sustainable Healthcare 2017 - Sensing in Surgery project.

\section{Acknowledgements}

We would like to thank the Erasmus MC and its staff (Gert-Jan Kleinrensink, Naomi Molenaar, Floris van Hoeckel, Floriaan Bruins, Karin Biermans) for helping to organise 
and to allow us to perform the cadaver study. We would also like to thank DePuy Synthes Johnson\&Johnson (Nazir-Ahmed Karbanee, Lukas Wagner, Jonathan Thompson) as our Sensing in Surgery grant collaborator and for the support, as well as allowing us to perform the experiments with their resources, such as the cadaver study.

\section{Author contributions}

Conceptualisation: BJB and TH; Formal Analysis: JCJW, WHAC, HJ, TSADH, WLAK; Funding acquisition: TH, BJB, JD; Investigation: WHAC, HJ, TSADH, WLAK, BJB; Methodology: WHAC, HJ, TSADH, WLAK, BJB; Project administration: JCJ, WHAC, HJ, TSADH, WLAK, TH; Software: WHAC, HJ, TSADH, WLAK; Supervision: JCJW, JD, TH; Validation: JCJ, TH, JD; Visualisation: JCJW, WHAC, HJ, TSADH, WLAK; Writing - original draft: JCJW, WHAC, HJ, TSADH, WLAK; Writing - review \& editing: JCJW, TH, JD.

\section{Erasmus Medical Centre institutional review board statement}

All total body donors were part of the national donor programme and have given written consent for tissue donation for educational and scientific purposes before passing away. Under Dutch law, and under these conditions no approval of the medical research ethics committee was required. Due to European privacy regulations, medical history was only available to the general practitioner of the donor and not to the receiving academic medical centre.

\section{Data availability statement}

The datasets generated and/or analysed from this study are available from the corresponding author on reasonable request.

\section{Conflict of interest}

Depuy Synthes Johnson\&Johnson funded the cadaver skillslab session and we carried out our experiment afterwards, using their equipment and tools. They are a collaborator of the Sensing in Surgery project. They did not have any influence on the results and conclusions of this paper. There are no conflicts of interest declared by all authors.

\section{References}

1. Landelijke Registratie Orthopedische Implantaten. (2019) Number of registered hip arthroplasties per year of surgery (20072018) in the lroi in April 2019. [Online]. Available: http://www.lroi-rapportage.nl/2-magazines-op-home-2019-numbers-registered-procedures-2007-2018

2. S. Giardini, D. Laney, A. Rutherford, and C. Farrar, "Monitoring femoral component insertion in cementless total hip arthroplasty, "Conference Proceedings of the Society for Experimental Mechanics Series, 012005.

3. G. Whitwell, C. L. Brockett, S. Young, M. Stone, and T. D. Stewart, "Spectral analysis of the sound produced during femoral broaching and implant insertion in uncemented total hip arthroplasty, "Proceedings of the Institution of Mechanical Engineers, Part H: Journal of Engineering in Medicine, vol. 227, no. 2, pp. 175-180, 2013.

4. L. C. Pastrav, S. V. N. Jaecques, I. Jonkers, G. V. D. Perre, and M. Mulier, "In vivo evaluation of a vibration analysis technique for the per-operative monitoring of the fixation of hip prostheses, “Journal of Orthopaedic Surgery and Research, vol. 4, no. 1, Sep 2009.

5. M. S. Rashid and R. Pullin, "The sound of orthopaedic surgery - the application of acoustic emission technology in orthopaedic surgery: a review, “European Journal of Orthopaedic Surgery \& Traumatology, vol. 24, no. 1, pp. 1-6, Jan 2014. [Online]. Available: https://doi.org/10.1007/s00590-012-1139-0

6. G. Qi, W. P. Mouchon, and T. E. Tan, "How much can a vibrational diagnostic tool reveal in total hip arthroplasty loosening? “Clinical Biomechanics, vol. 18, no. 5, pp. 444- 458, 2003. [Online]. Available: http://www.sciencedirect.com/science/article/pii/S0268003303000512

7. G. W. Rodgers, R. Welsh, L. J. King, A. J. Fitz Patrick, T. B. Woodfield, and G. J. Hooper, “Signal processing and event detection of hip implant acoustic emissions, "Control Engineering Practice, vol. 58, pp. 287 - 297, 2017.

8. A. J. Fitz Patrick, G. W. Rodgers, G. J. Hooper, and T. B. Woodfield, “Development and validation of an acoustic emission device to measure wear in total hip replacements in-vitro and in-vivo, "Biomedical Signal Processing and Control, vol. 33, pp. 281 288, 2017. [Online]. Available: http://www.sciencedirect.com/science/article/pii/S1746809416302233 
9. S. Oberst, J. Baetz, G. Campbell, F. Lampe, J. C. Lai, N. Hoffmann, and M. Morlock, “Vibro-acoustic and non-linear analysis of cadaveric femoral bone impaction in cavity preparations, "International Journal of Mechanical Sciences, vol. 144, pp. 739 - 745, 2018.

10. P. H. M. Pechon, R. Pullin, M. J. Eaton, S. A. Jones, and S. Evans, “Acoustic emission technology can warn of impending iatrogenic femur fracture during femoral canal preparation for uncemented hip replacement. a cadaveric animal bone study, "Journal of Medical Engineering \& Technology, vol. 42, no. 2, pp. 72-87, 2018.

11. N. Gueiral and E. Nogueira, Recent Advances in Arthroplasty. In Tech, 2011, ch. 8, pp. 131 - 146.

12. M. Mavrogordato, M. Taylor, A. Taylor, and M. Browne, "Realtime monitoring of progressive damage during loading of a simplified total hip stem construct using embedded acoustic emission sensors, “Medical Engineering \& Physics, vol. 33, no. 4, pp. $395-406,2011$.

13. M. Mulier, C. Pastrav, and G. Van der Perre, "Per-operative vibration analysis: a valuable tool for defining correct stem insertion: preliminary report, “Ortop Traumatol Rehabil, vol. 10, no. 6, pp. 576-82, 2008.

14. C. P. Childers and M. Maggard-Gibbons, "Understanding costs of care in the operating room”, JAMA Surgery, vol. 153, no. 4, pp. e176 233-e176 233, 042018.

15. M. Grimm, Standard Handbook of Biomedical Engineering and Design. New York, United States: McGraw-Hill, 2003, ch. Orthopedic Biomaterials, pp. 15.1-15.22.

16. N. Shibanuma, Y. Hata, T. Nishiyama, T. Fujishiro, H. Tateishi, and M. Kurosaka, “Determination of total hip arthroplasty stem stability by intraoperative measurement using an acoustic testing technique," in Proc. of the 54th Annual Meeting of the Orthopaedic Research Society, 2008, p. 1842.

17. I. Morohashi, H. Iwase, A. Kanda, T. Sato, Y. Homma, A. Mogami, O. Obayashi, and K. Kaneko, "Acoustic pattern evaluation during cementless hip arthroplasty surgery may be a new method for predicting complications, “SICOTJ, vol. 3, no. 13, 2017.

18. A. Crisman, N. Yoder, M. McCuskey, R. Meneghini, and P. Cornwell, "Femoral component insertion monitoring using human cadaveric specimens, "Conference Proceedings of the Society for Experimental Mechanics Series, 012007.

19. "CES Edupack Software," Cambridge, UK, 1999 - 2019.

20. J. O'Connor, L. Borges, F. Duda, and A. da Cruz, "Growth and reabsorption in biological tissues. xxxvi ibero-latin American congress on computational methods in engineering (cilamce2015)," 112015.

21. "Eigenfrequency analysis." [Online]. Available: https://www.comsol.com/multiphysics/eigenfrequency-analysis

22. Circle Health. (2018) Total hip replacement surgery. [Online]. Available: https://www.youtube.com/watch?v=CVAPmdBC1p0

23. L. Mosekilde, "Sex differences in age-related loss of vertebral trabecular bone mass and structure-biomechanical consequences, "Bone, vol. 10, no. 6, pp. 425 - 432,1989. [Online]. Available: http://www.sciencedirect.com/science/article/pii/8756328289900744

24. "Actis surgical technique," http://synthes.vo.llnwd.net/o16/LLNWMB8/US\%20Mobile/Synthes\%20North\%20America/Product\%20Support\%20Materials/Technique\%20Guides/Actis\%20Surgical\%20Technique\%20March\%202018.pdf, Warsaw, IN 46582 USA, 2017.

25. Purves D, Augustine GJ, Fitzpatrick D, et al., editors. Neuroscience. 2nd edition. Sunderland (MA): Sinauer Associates; 2001. The Audible Spectrum. Available from: https://www.ncbi.nlm.nih.gov/books/NBK10924/ 